\title{
Long-Range Nematic Order in Two-Dimensional Active Matter
}

\author{
Benoît Mahault $\oplus^{1}$ and Hugues Chaté $\oplus^{2,3}$ \\ ${ }^{1}$ Max Planck Institute for Dynamics and Self-Organization (MPIDS), 37077 Göttingen, Germany \\ ${ }^{2}$ Service de Physique de l'Etat Condensé, CEA, CNRS Université Paris-Saclay, CEA-Saclay, 91191 Gif-sur-Yvette, France \\ ${ }^{3}$ Computational Science Research Center, Beijing 100193, China
}

(Received 16 April 2021; accepted 14 June 2021; published 23 July 2021)

\begin{abstract}
Working in two space dimensions, we show that the orientational order emerging from self-propelled polar particles aligning nematically is quasi-long-ranged beyond $\ell_{r}$, the scale associated to induced velocity reversals, which is typically extremely large and often cannot even be measured. Below $\ell_{r}$, nematic order is long-range. We construct and study a hydrodynamic theory for this de facto phase and show that its structure and symmetries differ from conventional descriptions of active nematics. We check numerically our theoretical predictions, in particular the presence of $\pi$-symmetric propagative sound modes, and provide estimates of all scaling exponents governing long-range space-time correlations.
\end{abstract}

DOI: 10.1103/PhysRevLett.127.048003

Studies of active matter continue to flourish, exploring more and more complex situations in an increasingly quantitative manner (For recent experimental works, see, e.g., [1-11]). Evidence has accumulated that shows active matter exhibits properties that are impossible in thermal equilibrium or even in driven systems (For recent theoretical works, see, e.g., [12-25]). In spite of all this progress, important fundamental questions remain open. Such a longstanding issue is whether true long-range nematic order can emerge in two space dimensions (2D).

Whereas it is now well known, notably thanks to the seminal work by Toner and Tu, that long-range polar order can arise in 2D active systems [26-34], the debate has remained open for active nematics: On the one hand, theoretical results conclude that nematic order can at best be quasi-long-range [35], as in equilibrium, albeit with important differences [37-43]. On the other hand, numerical and experimental results obtained on self-propelled particles without spontaneous velocity reversals yielded convincing data demonstrating true long-range nematic order over a large range of scales $[44,45]$.

In this Letter, we study 2D dry dilute active nematicsthe framework in which the question of the asymptotic nature of nematic order was mostly discussed-using numerical simulations and theory. We show that the homogeneous ordered phase of a Vicsek-style model of polar self-propelled particles aligning nematically actually

Published by the American Physical Society under the terms of the Creative Commons Attribution 4.0 International license. Further distribution of this work must maintain attribution to the author(s) and the published article's title, journal citation, and DOI. Open access publication funded by the Max Planck Society. displays true long-range nematic order only up to $\ell_{r}$, the scale associated with the typical time between velocity reversals induced by collisions and noise. Beyond $\ell_{r}$, global nematic order decays algebraically with system size, in agreement with general theoretical arguments. However $\ell_{r}$ can easily take astronomically large values such that there exists a region of parameter space in which only true long-range nematic order can be observed. We derive a hydrodynamic theory for this regime and show that it possesses a structure and symmetries different from those of standard active nematics. Our analysis of this field theory predicts $\pi$-symmetric propagative sound modes and the scaling form of space-time fluctuations. Finally, numerical results confirm the theory and allow us to estimate all scaling exponents.

We use the Vicsek-style model of polar particles with nematic alignment first introduced in [44]. Particles $i=$ $1, \ldots, N$ evolve at discrete time steps with constant speed $v_{0}$ in square domains of linear size $L$ with periodic boundary conditions, interacting with neighbors within unit distance. Their positions $\mathbf{r}_{i}$ and unit-length orientations $\mathbf{e}_{i}=\mathbf{e}\left(\theta_{i}\right)$ obey

$$
\begin{aligned}
\mathbf{r}_{i}^{t+1} & =\mathbf{r}_{i}^{t}+v_{0} \mathbf{e}_{i}^{t+1}, \\
\mathbf{e}_{i}^{t+1} & =\left(\mathcal{R}_{\eta} \circ \vartheta\right)\left\langle\operatorname{sign}\left[\mathbf{e}_{i}^{t} \cdot \mathbf{e}_{j}^{t}\right] \mathbf{e}_{j}^{t}\right\rangle_{j \sim i},
\end{aligned}
$$

where $\vartheta$ normalizes vectors $(\vartheta(\mathbf{u})=\mathbf{u} /\|\mathbf{u}\|),\langle\cdot\rangle_{j \sim i}$ denotes the average over neighbors $j$ of $i$ including $i$, and $\mathcal{R}_{\eta}$ rotates them by a random angle drawn from a uniform distribution in $[-\pi \eta, \pi \eta]$, independently for every particle at every time step. The two main parameters are the global density $\bar{\rho}=N / L^{2}$ and the noise strength $\eta$. The phase diagram in the $(\bar{\rho}, \eta)$ plane is typical of Vicsek-style models [34]. 
All results presented below were obtained with $v_{0}=0.5$ and $\bar{\rho}=2$.

We focus on the homogeneous nematic liquid that exists for $\eta \lesssim 0.21$, where the time and particle averaged nematic order parameter $S=\left\langle\left|\left\langle e^{i 2 \theta_{k}^{t}}\right\rangle_{k}\right|\right\rangle_{t}$ takes $\mathcal{O}(1)$ values. In this state, particles can be split into two "polar" subpopulations according to which of the two opposite directions defined by the nematic order their orientation is closest. The nematic interaction in Eq. (1b) aligns particles belonging to the same population and antialigns particles belonging to opposite populations, so that particles mostly stay in the same population. Nevertheless, under the action of interactions and noise, they can eventually turn enough that they join the other population. It was shown in [44] that the distance traveled between such reversals is distributed exponentially with a characteristic length $\ell_{r}$ independent of system size. In Fig. 1(a), we show that $\ell_{r}$ grows very fast when the noise strength $\eta$ decreases. A good fit of our data is that $\ell_{r} \sim \eta^{-8}$.

In [44], the global nematic order parameter $S$ was found to decrease slower than a power of $L$ and consistent with an algebraic decay to a finite asymptotic value $\left[S(L)-S(\infty) \sim L^{-\varpi}\right]$. These results led to conclude to true long-range nematic order, but they were obtained on a range of system sizes barely encompassing $\ell_{r}$. Here, choosing a noise strength such that $\ell_{r}$ is not too large, we find that for $L>\ell_{r}, S$ decays like a small power of $L$, in departure from the $L<\ell_{r}$ behavior [Fig. 1(b)]. Asymptotically, nematic order is only quasi-long-range, in agreement with standard theories [42].

Nevertheless, in most of the homogeneous nematic phase, $\ell_{r}$ is so large that only the $L<\ell_{r}$ regime is accessible and it is thus important to study it per se. Working in this regime, we confirm that nematic order appears as if fully longrange; moreover, the scaling of the local slope $\sigma(L) \equiv$ $-d \ln (S) / d \ln (L) \sim L^{-\varpi}$ allows us to identify an internal crossover scale $\ell_{\mathrm{c}}$ separating two regimes with different values of $\varpi$ [Fig. 1(c)]. (a) $\ell_{r}$

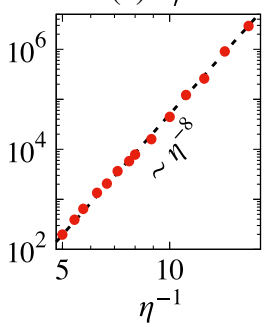

(b) $S(L)$

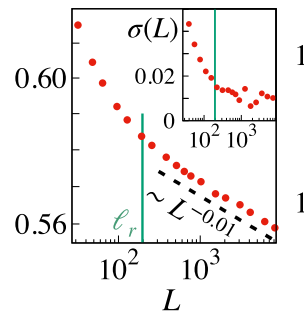

(c) $\sigma(L)$

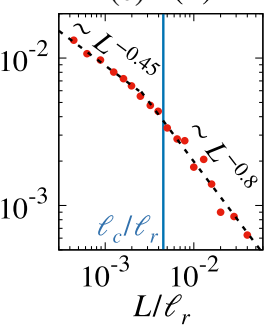

FIG. 1. Vicsek-style model (1) $\left(v_{0}=0.5, \bar{\rho}=2\right)$. (a) Variation of $\ell_{r}$ with $\eta$. (b) Global nematic order $S$ vs linear system size $L$ (in $\log$ scales); for $L<\ell_{r} \simeq 200, S$ decreases slower than a power law in a manner compatible with long-range order, while a slow algebraic decay is observed for $L \gg \ell_{r}(\eta=0.2)$; inset: local slope $\sigma(L)$ showing a plateau for $L \gg \ell_{r}$. (c) $\sigma(L)$ vs $L / \ell_{r}$ in the long-range ordered regime ( $\eta=0.1$, for which $\left.\ell_{r} \simeq 50000\right)$; note the crossover scale $\ell_{\mathrm{c}} \simeq 200$ separating two scaling regimes.
We now present a theory of the long-range-ordered nematics present on scales much smaller than $\ell_{r}$. Full details of calculations are given in [46]. Our approach is rooted in the observation that a fluctuation in the form of a small polar packet propagates along one of the two directions set by the mean order due to the persistence in the average particle motion on scales below $\ell_{r}$. This implies that a theory capturing the fate of fluctuations on such scales cannot be invariant by reflexion along order, in contrast with usual treatments of the fast reversal limit $[34,38,40,42]$. We thus directly consider two populations, $R$ and $L$, of polar active particles with speed $v_{0}$ aligning their velocity with neighbors if those belong to the same population, and antialign it otherwise. This is not equivalent to usual nematic alignment: two particles of the same population will align even if their relative angle is obtuse, and they will antialign if they belong to different populations, irrespective of their angle. We further assume that the populations exchange members randomly at rate $1 / \tau_{r} \simeq \ell_{r} / v_{0}$ [48]. We first write Boltzmann equations ruling the evolution of the one-body probability density functions $f_{L}(\mathbf{r}, \theta, t)$ and $f_{R}(\mathbf{r}, \theta, t)$ :

$\partial_{t} f_{L}+\mathbf{v}(\theta) \cdot \nabla f_{L}=\frac{1}{\tau_{r}}\left(f_{R}-f_{L}\right)+I_{\mathrm{sd}}\left[f_{L}\right]+I_{\mathrm{co}}\left[f_{L}, f_{R}\right]$,

and the equation governing $f_{R}$ is given by swapping the $L$ and $R$ subscripts. In (2), $\mathbf{v}(\theta)=v_{0} \mathbf{e}(\theta)$ is the velocity of particles with orientation $\theta$, whereas the integrals $I_{\text {sd }}$ and $I_{\text {co }}$, given in [46], describe the effects of angular self-diffusion and collisions.

Introducing the more convenient $f=f_{R}+f_{L}$ and $g=f_{R}-f_{L}$, expanding $f$ and $g$ in Fourier series of $\theta$ (e.g., $\left.f(\mathbf{r}, \theta, t)=(1 / 2 \pi) \sum_{k=-\infty}^{+\infty} f_{k}(\mathbf{r}, t) e^{-i k \theta}\right)$, the Boltzmann equations are dedimensionalized and transformed into a hierarchy of partial differential equations for the $f_{k}$ and $g_{k}$ fields. As shown in [46], a linear stability analysis of the disordered solution $\rho \equiv f_{0}=\bar{\rho}$ (the total density), $f_{k>0}=g_{k}=0$ reveals that it is unstable to $g_{1}$ perturbations at large density and/or weak noise. The field $g_{1}$ is thus responsible for the onset of orientational order. Note that $g_{1}$ measures polar order within each population, i.e., is a proxy for global nematic order. The equations for $\rho$ and $g_{0}$ read

$$
\begin{aligned}
\partial_{t} \rho & =-\operatorname{Re}\left[\nabla^{*} f_{1}\right], \\
\partial_{t} g_{0} & =-2 \tau_{r}^{-1} g_{0}-\operatorname{Re}\left[\nabla^{*} g_{1}\right],
\end{aligned}
$$

where $\nabla \equiv \partial_{x}+i \partial_{y}$ denotes the complex gradient.

Following the Boltzmann-Ginzburg-Landau approach [34,49-51], one can build step by step a scaling ansatz using a small parameter $\varepsilon$ marking the magnitude of order near onset $\left(\left|g_{1}\right| \sim \varepsilon\right)$. As detailed in [46], this leads to 
$\left|g_{k \geq 1}\right| \sim \varepsilon^{k},\left|f_{k>1}\right| \sim \varepsilon^{k}$, and $\partial_{t} \sim \nabla \sim \varepsilon$ [52]. In addition, considering Eqs. (3a) and (3b), one completes the scaling ansatz by $\left|g_{0}\right| \sim \varepsilon,|\delta \rho| \sim\left|f_{1}\right| \sim \mathcal{E}^{2}$. Truncating and closing the Boltzmann hierarchy at order $\varepsilon^{4}$ yields hydrodynamic equations for $f_{1}$ and $g_{1}$ :

$$
\begin{aligned}
\partial_{t} f_{1}= & -\frac{1}{2} \nabla \rho+\left(\mu[\rho]-\zeta\left|g_{1}\right|^{2}\right) f_{1}+D_{f} \Delta f_{1} \\
& +\left(\alpha\left[g_{0}\right]-\chi_{1} g_{0}\left|g_{1}\right|^{2}-\chi_{2} f_{1}^{*} g_{1}\right) g_{1}+D_{g} g_{0} \Delta g_{1} \\
& +\kappa_{1}[\rho] \nabla^{*} g_{1}^{2}+\kappa_{2}[\rho] g_{1}^{*} \nabla g_{1}+\kappa_{3}\left(\nabla^{*} g_{0}\right)\left(\nabla g_{1}\right) \\
\partial_{t} g_{1}= & -\frac{1}{2} \nabla g_{0}+\left(\nu[\rho]-\Gamma[\rho]\left|g_{1}\right|^{2}\right) g_{1}+\Omega[\rho] \Delta g_{1} \\
& -\sigma g_{1}^{2} \nabla^{*} g_{0}+\beta\left[g_{0}\right] f_{1}+\lambda_{1} g_{0} \nabla^{*} g_{1}^{2}+\lambda_{2} g_{0} g_{1}^{*} \nabla g_{1} \\
& +\lambda_{3} g_{1}^{*} \nabla f_{1}+\lambda_{4} \nabla^{*}\left(g_{1} f_{1}\right)+\lambda_{5} f_{1}^{*} \nabla g_{1},
\end{aligned}
$$

where all coefficients depend on the particle-level parameters $\bar{\rho}, \eta$, and $\tau_{r}$. (see [46] for their explicit expressions), and local dependencies on $\rho$ and $g_{0}$ are indicated.

Equations (3) are structurally different from hydrodynamic theories written for active nematics [34,38,40,42], as seen by the many advective and nonlinear terms. A consequence of the $2 \pi$ symmetry of the interaction between our polar particles is that the theory above does not explicitly include the usual nematic order parameter $f_{2}$ [50]. However, as detailed in [46] the scaling ansatz used for the derivation of (3) leads to $f_{2} \sim g_{1}^{2}$, such that $g_{1}$ effectively measures local nematic order.

The pairs of equations for $\left(\rho, f_{1}\right)$ and $\left(g_{0}, g_{1}\right)$ resemble two coupled Toner-Tu (TT) systems. Both $\rho$ and $g_{0}$ are advected by the corresponding order fields $f_{1}$ and $g_{1}$, which are not $\pi$ symmetric. Discarding the couplings to $\rho$ and $f_{1}$, Eqs. (3b) and (3d) are almost like the TT equations in the limit $\tau_{r} \rightarrow \infty$. They however are missing the terms $\sim g_{0} g_{1}$ and $\sim g_{1} \nabla g_{1}$ that are forbidden by the $R \leftrightarrow L$ symmetry of the problem, which imposes the equations to be invariant under $g \leftrightarrow-g$.

Equations (3), even if formally derived at the onset of order, reflect the symmetries of the deeply ordered phase. We now focus on fluctuations in that phase, i.e., around the homogeneous ordered solution $\rho=\bar{\rho}, g_{0}=f_{1}=0$, $g_{1}=\bar{g} \equiv \sqrt{\nu[\bar{\rho}] / \Gamma[\bar{\rho}]}$ that exists when $\nu[\bar{\rho}]>0 \quad[53]$. Linearizing Eqs. (3) around this solution, separating parallel $(\|)$ and transverse $(\perp)$ components, we obtain a system of six equations governing small perturbations $\delta \rho$, $\delta g_{0}, \delta f_{\|}, \delta f_{\perp}, \delta g_{\|}$, and $\delta g_{\perp}$. We find that $\delta \rho$ and $\delta g_{\perp}$ are hydrodynamic modes, while $\delta f_{\|}, \delta f_{\perp}$, and $\delta g_{\|}$decay rapidly. Since $\tau_{r}$ can take arbitrary large values, we also consider $\delta g_{0}$ as hydrodynamic. Enslaving the fast modes, we obtain the following linear system:

$$
\begin{aligned}
\partial_{t} \delta \rho= & \left(D_{\rho \|} \partial_{\|\|}^{2}+D_{\rho \perp} \partial_{\perp \perp}^{2}\right) \delta \rho-\lambda_{0} \partial_{\|} \delta g_{0} \\
& +D_{\rho g} \partial_{\| \perp}^{2} \delta g_{\perp}+\eta_{1} \partial_{\| t}^{2} \delta g_{0}, \\
\partial_{t} \delta g_{0}= & \left(D_{0 \|} \partial_{\|\|}^{2}+D_{0 \perp} \partial_{\perp \perp}^{2}-2 \tau_{r}^{-1}\right) \delta g_{0} \\
& -\kappa_{0} \partial_{\|} \delta \rho-v_{0} \partial_{\perp} \delta g_{\perp}+\eta_{2} \partial_{\| t}^{2} \delta \rho, \\
\partial_{t} \delta g_{\perp}= & \left(D_{\|} \partial_{\|\|}^{2}+D_{\perp} \partial_{\perp \perp}^{2}\right) \delta g_{\perp} \\
& +\gamma \partial_{\| \perp}^{2} \delta \rho-\alpha_{0} \partial_{\perp} \delta g_{0}+\eta_{3} \partial_{\perp t}^{2} \delta g_{0},
\end{aligned}
$$

where we split the complex gradient into $\nabla=\partial_{\|}+i \partial_{\perp}$, and all the (bare) coefficients are given in [46,54].

We first note that in the small $\tau_{r}$ limit, such that particles reverse their orientation many times on the scale at which we observe fluctuations, $\delta g_{0}$ is nonhydrodynamic [cf. Eq. (4b)]. Equations (4) then reduce to those of an homogeneous active nematic (with $\delta g_{\perp}$ playing the role of the transverse fluctuations of nematic order, see $[42,46])$.

In the $\tau_{r} \rightarrow \infty$ limit of main interest here, on the other hand, we neglect the term $2 \tau_{r}^{-1} \delta g_{0}$ in Eq. (4b). To compute space and time correlation functions of the three hydrodynamic fields $\delta \rho, \delta g_{0}$, and $\delta g_{\perp}$, we equip Eqs. (4) with additive, uncorrelated, zero-mean noise terms. For Eq. (4a), governing density fluctuations, this noise is conserved and we write it $\partial_{\|} h_{\rho \|}+\partial_{\perp} h_{\rho \perp}$. Writing the (stochastic) Eqs. (4) in Fourier space, taking the long wavelength, low frequency limit $q, \omega \rightarrow 0$, rather tedious but standard calculations detailed in [46] lead to

$$
\begin{aligned}
& \left\langle|\delta \hat{\rho}(\omega, \boldsymbol{q})|^{2}\right\rangle \underset{\omega, q \rightarrow 0}{\simeq} \mathcal{D}(\omega, \boldsymbol{q})^{-1}\left[\left(q_{\|}^{2} \Delta_{\rho \|}+q_{\perp}^{2} \Delta_{\rho \perp}\right)\left(\omega^{2}-v_{0} \alpha_{0} q_{\perp}^{2}\right)^{2}+\Delta_{0} q_{\|}^{2} \lambda_{0}^{2} \omega^{2}+\Delta_{\perp}\left(v_{0} \lambda_{0}\right)^{2} q_{\|}^{2} q_{\perp}^{2}\right], \\
& \left\langle\left|\delta \hat{g}_{0}(\omega, \boldsymbol{q})\right|^{2}\right\rangle \underset{\omega, q \rightarrow 0}{\simeq} \mathcal{D}(\omega, \boldsymbol{q})^{-1}\left[\left(q_{\|}^{2} \Delta_{\rho \|}+q_{\perp}^{2} \Delta_{\rho \perp}\right) \kappa_{0}^{2} q_{\|}^{2} \omega^{2}+\Delta_{0} \omega^{4}+\Delta_{\perp} v_{0}^{2} q_{\perp}^{2} \omega^{2}\right], \\
& \left\langle\left|\delta \hat{g}_{\perp}(\omega, \boldsymbol{q})\right|^{2}\right\rangle \underset{\omega, q \rightarrow 0}{\simeq} \mathcal{D}(\omega, \boldsymbol{q})^{-1}\left[\left(q_{\|}^{2} \Delta_{\rho \|}+q_{\perp}^{2} \Delta_{\rho \perp}\right)\left(\alpha_{0} \kappa_{0}\right)^{2} q_{\|}^{2} q_{\perp}^{2}+\Delta_{0} \alpha_{0}^{2} q_{\perp}^{2} \omega^{2}+\Delta_{\perp}\left(\omega^{2}-\kappa_{0} \lambda_{0} q_{\|}^{2}\right)^{2}\right],
\end{aligned}
$$

where $\Delta_{\rho \|}$ and $\Delta_{\rho \perp}$ are the amplitudes of the conserved $\rho$ noise, $\Delta_{0}$ and $\Delta_{\perp}$ those of the $g_{0}$ and $g_{\perp}$ noises, and

$$
\mathcal{D}(\omega, \boldsymbol{q}) \equiv\left|\omega-i \varepsilon_{d}(\boldsymbol{q})\right|^{2} \times\left|\omega-c\left(\theta_{\boldsymbol{q}}\right) q+i \varepsilon_{p}(\boldsymbol{q})\right|^{2} \times\left|\omega+c\left(\theta_{\boldsymbol{q}}\right) q+i \varepsilon_{p}(\boldsymbol{q})\right|^{2} .
$$


As shown in [46], where their explicit forms are given, $\varepsilon_{d, p}(\boldsymbol{q}) \sim q^{2}$, whereas the anisotropic speed is

$$
c\left(\theta_{\boldsymbol{q}}\right)=\sqrt{\kappa_{0} \lambda_{0} \cos ^{2}\left(\theta_{\boldsymbol{q}}\right)+v_{0} \alpha_{0} \sin ^{2}\left(\theta_{\boldsymbol{q}}\right)},
$$

where $\theta_{\boldsymbol{q}}$ denotes the angle between $\boldsymbol{q}$ and the mean order. Equations (5), (6), and (7) are fundamentally different from their counterparts in both active nematics and the TT class: at most orientations $\theta_{q}$ correlations have a diffusive peak and two symmetric propagative peaks at $\omega= \pm c\left(\theta_{q}\right) q$.

Equal-time correlation functions are easily obtained by integrating Eqs. (5) over $\omega$. They all diverge as $q^{-2}$ for most $\theta_{q}$, which means that nematic order is only quasi-longrange at this linear level, a situation similar to that of polar order in TT theory. To resolve this marginal situation, one needs to study nonlinear hydrodynamics. We first repeat the calculations leading to Eqs. (4) keeping the leading order nonlinearities (in fields and gradients). The structure of our theory shares similarities with the polar case. We thus limit ourselves to terms of order 3 in fields and gradients [29]. After lengthy but straightforward manipulations (detailed in [46]), we obtain

$$
\begin{aligned}
\partial_{t} \delta \rho= & \mathcal{L}_{\rho}+j_{1} \partial_{\|}\left(\delta g_{0} \delta \rho\right)+j_{2} \partial_{\perp}\left(\delta g_{0} \delta g_{\perp}\right), \\
\partial_{t} \delta g_{0}= & \mathcal{L}_{g_{0}}+c_{1} \partial_{\|} \delta \rho^{2}+c_{2} \partial_{\|} \delta g_{\perp}^{2}+c_{3} \partial_{\|} \delta g_{0}^{2}, \\
\partial_{t} \delta g_{\perp}= & \mathcal{L}_{g_{\perp}}+w_{1} \delta g_{\perp} \partial_{\|} \delta g_{0}+w_{2} \delta g_{0} \partial_{\|} \delta g_{\perp} \\
& +w_{3} \delta g_{0} \partial_{\perp} \delta \rho+w_{4} \delta \rho \partial_{\perp} \delta g_{0}+w_{5} \delta g_{\perp} \partial_{t} \delta \rho,
\end{aligned}
$$

where $\mathcal{L}$ is the linear part [Eqs. (4)]. Introducing the scaling exponents via $x_{\perp} \rightarrow b x_{\perp}, x_{\|} \rightarrow b^{\xi} x_{\|}, t \rightarrow b^{z} t$, $\delta g_{\perp} \rightarrow b^{\chi} \delta g_{\perp}, \delta g_{0} \rightarrow b^{\chi_{0}} \delta g_{0}, \delta \rho \rightarrow b^{\chi_{\rho}} \delta \rho$ and imposing a fixed point condition on Eqs. (8) considered valid in any dimension $d$ yields the following values of the exponents

$$
z=2, \quad \xi=1, \quad \chi=\chi_{0}=\chi_{\rho}=1-\frac{d}{2} \text { (linear level). }
$$

We thus have isotropic $(\xi=1)$ diffusive $(z=2)$ scaling with quasi-long-range order in $d=2(\chi=0)$ at the linear level, as for both active nematics and TT theory.

At the linear fixed point, nine of the ten nonlinear terms in Eqs. (8) scale like $b^{(4-d) / 2}$, i.e., are relevant in $d \leq d_{c} \equiv 4$ (the exception is $\omega_{5}$ ). This means that the linear theory breaks down in $d \leq 4$, and that we should in principle embark on a complete renormalization group analysis to obtain exponent values. We leave this challenging task for future studies. Instead we rely on general considerations and formal similarities with TT theory to make predictions that we test numerically.

Replacing the eigenfrequencies $\omega=c\left(\theta_{\boldsymbol{q}}\right) q$ into Eqs. (4a) and (4b) (expressed in Fourier space), we find at leading order $\quad c\left(\theta_{\boldsymbol{q}}\right) q \delta \hat{\rho} \sim q_{\|} \delta \hat{g}_{0} \quad$ and $\quad c\left(\theta_{\boldsymbol{q}}\right) q \delta \hat{g}_{0} \sim q_{\|} \delta \hat{\rho}+q_{\perp} \delta \hat{g}_{\perp}$.
Therefore, taking $\theta_{\boldsymbol{q}}=\pi / 2$ we get that $\left|\delta \hat{g}_{0}\right| \sim\left|\delta \hat{g}_{\perp}\right|$, such that $\chi_{0}=\chi$, while for any orientation of $\boldsymbol{q}$ not purely longitudinal or transverse we have $|\delta \hat{\rho}| \sim\left|\delta \hat{g}_{0}\right|$, which implies $\chi_{\rho}=\chi_{0}$. It is thus likely that the equality $\chi=\chi_{0}=\chi_{\rho}$ holds even at the nonlinear level.

Given that the structure of Eqs. (8) is similar to that found in TT theory, we follow [29] and conjecture that the scaling of correlation functions in the nonlinear theory is obtained using renormalized noise coefficients $\tilde{\Delta}=q_{\perp}^{z-\zeta} f_{\Delta}\left(q_{\|} / q_{\perp}^{\xi}\right)$ and renormalized dampings $\tilde{\varepsilon}=q_{\perp}^{z} f_{\varepsilon}\left(q_{\|} / q_{\perp}^{\xi}\right)$ (where we have defined $\zeta \equiv d-1+2 \chi+\xi)$, with functions $f_{\Delta}$ and $f_{\varepsilon}$ expected to be universal and to satisfy $f_{\Delta, \varepsilon}(x) \underset{x \rightarrow 0}{\longrightarrow}$ const, $f_{\Delta}(x) \underset{x \rightarrow \infty}{\longrightarrow} x^{(z-\zeta) / \xi}$, and $f_{\varepsilon}(x) \underset{x \rightarrow \infty}{\longrightarrow} x^{z / \xi}$. On the other hand the speeds $c\left(\theta_{\boldsymbol{q}}\right)$ should not be renormalized. Under all these assumptions, it is possible to predict the asymptotic behavior of equal-time correlation functions. For instance

$$
\left\langle\left|\delta \hat{g}_{\perp}\left(q_{\|}\right)\right|^{2}\right\rangle \underset{q_{\|} \rightarrow 0}{\sim} q_{\|}^{-\zeta / \xi}, \quad\left\langle\left|\delta \hat{g}_{\perp}\left(q_{\perp}\right)\right|^{2}\right\rangle_{q_{\perp} \rightarrow 0}^{\sim} q_{\perp}^{-\zeta} .
$$

(For other functions, see [46].)

We now come back to our Vicsek-style model at noise strength $\eta=0.1$ and show data for the order correlations confirming the structure of the above theory and providing estimates of the scaling exponents. Additional results for the densities $\rho$ and $g_{0}$ will be published elsewhere [55]. We actually measure the transverse nematic order $\delta Q_{\perp}$, which, when aligned along the horizontal direction and assuming small angular deviations, is a good proxy of $\delta g_{\perp}$. $\left(\delta Q_{\perp} \sim \cos (\theta) \sin (\theta) \sim \delta \theta \sim \bar{\rho}^{-1} \delta g_{\perp}\right)$.

The frequency spectra do have the qualitative structure predicted by Eqs. (5): two symmetric propagative peaks and a central diffusive one [Fig. 2(a)]. As expected, peak locations, at a fixed angle $\theta_{q}$ are proportional to $q$, allowing the easy measurement of the sound speed $c\left(\theta_{\boldsymbol{q}}\right)$, which we find in perfect quantitative agreement with Eq. (7) [Fig. 2(b)]. Peak widths provide estimates of $z$ and $z / \xi$ in the $\perp$ and $\|$ directions, as in TT theory. As shown in Fig. 2(c), we find a crossover at the same scale $\ell_{\mathrm{c}}$ as observed in Fig. 1(c). For scales below $\ell_{\mathrm{c}}$ we find $z^{\prime} \simeq 1.75$ and $z^{\prime} / \xi^{\prime} \simeq 1.4$, while we are only able to estimate $z / \xi \simeq 1.1$ in the asymptotic regime (we use primes to denote exponent values measured below $\ell_{\mathrm{c}}$ ). The equaltime order correlation function shown in Fig. 2(c) in the $\|$ and $\perp$ directions, also exhibits a crossover at $\ell_{\mathrm{c}}$. From the precrossover scaling we estimate $\zeta^{\prime} \simeq 1.75$ and $\zeta^{\prime} / \xi^{\prime} \simeq 1.4$, while we find $\zeta / \xi \simeq 1.1$ in the $q<2 \pi / \ell_{\mathrm{c}}$ regime.

We thus have two sets of scaling exponents: for scales below $\ell_{\mathrm{c}}$, the above estimates lead to $z^{\prime}=\zeta^{\prime} \simeq 1.4, \xi^{\prime} \simeq 1.25$, and $2 \chi^{\prime} \simeq-0.5$. Note that this yields $-2 \chi^{\prime} / \xi^{\prime} \simeq 0.4$, in agreement with our estimate of $\varpi^{\prime} \simeq 0.45$ in Fig. 1(c) [56]. For scales beyond $\ell_{\mathrm{c}}$, we have $z=\zeta$, but cannot estimate $\xi$ from correlation functions. Using $\varpi \simeq 0.8$ [Fig. 1(c)], yields $\xi \simeq 1.1$ and $2 \chi \simeq-0.9$, and finally $z=\zeta \simeq 1.2$. 

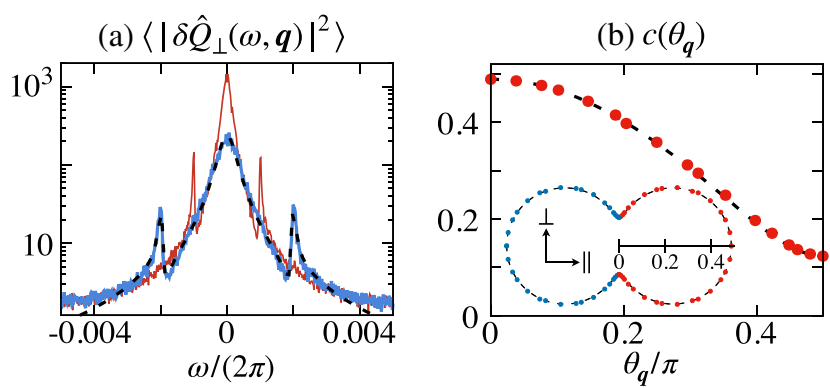

(c) peaks width $\varepsilon_{\mathrm{p}}(\boldsymbol{q})$
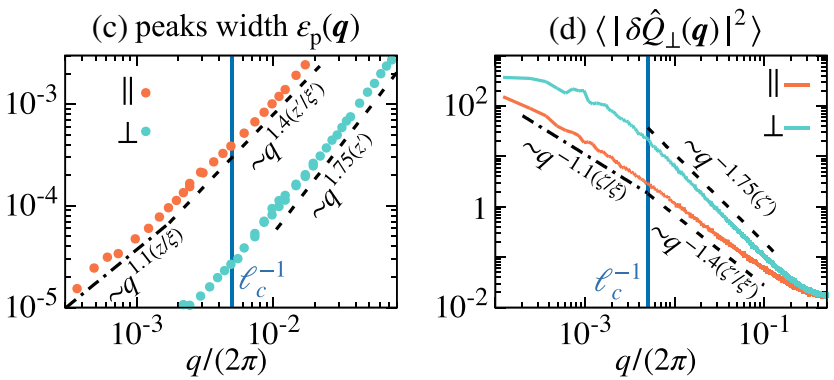

FIG. 2. Space-time correlations of fluctuations in the $L \ll \ell_{r}$ regime $(\eta=0.1, L=8192)$. (a) Frequency spectra of order fluctuations at angle $\theta_{\boldsymbol{q}}=(\pi / 4)$ with $(q / 2 \pi)=0.002$ (red) and 0.004 (blue). The black dashed line is a fit by the theoretical predictions of Eqs. (5). (b) Angular dependence of the measured (dots) and predicted (dashed line) sound speed $c\left(\theta_{\boldsymbol{q}}\right)$; Inset: polar plot showing the $\pi$ symmetry of $c\left(\theta_{q}\right)$. (c),(d) Scaling vs $q$ in the $\|$ and $\perp$ directions of frequency peak widths [measured from fits as shown in panel (a)] (c) and equal-time order correlation function (d).

A few remarks are in order: (i) both below and above $\ell_{\mathrm{c}}$, $z=\zeta=1+2 \chi+\xi$, a hyperscaling relation also verified by polar flocks that implies that the dominant noises are additive and their amplitude is not renormalized [31]; (ii) in our nematic phase the anisotropy exponent $\xi \simeq 1.1>1$, at odds with 2D polar flocks for which $\xi \simeq 0.95<1$ [31], but in both cases we cannot exclude that scaling is asymptotically isotropic.

To summarize, the orientational order emerging from self-propelled polar particles aligning nematically is always quasi-long-range asymptotically, but this regime is only observed beyond $\ell_{r}$, the scale associated with induced velocity reversals, which can easily take very large values and often cannot even be measured. Below $\ell_{r}$, nematic order appears fully long-range. Constructing a hydrodynamic theory from microscopic grounds, we showed that this de facto phase has a structure and symmetries distinct from both conventional descriptions of active nematics and Toner and Tu theory. Consequently, systems in the corresponding class exhibit features never reported so far, such as long-range nematic order and the presence $\pi$-symmetric propagative sound modes.

Finally, we believe our findings can be observed experimentally, as long as the rate of velocity reversals, be they induced or spontaneous, is small. After all, nematic alignment resulting from inelastic collisions between elongated objects is quite generic. Confined bacteria and motility assays are promising systems in this regard.

We thank Xia-qing Shi and Alexandre Solon for a critical reading of this manuscript. We acknowledge generous allocations of cpu time on the Living Matter Department cluster in MPIDS, and on Beijing CSRCs Tianhe supercomputer.

[1] V. Soni, E. S. Bililign, S. Magkiriadou, S. Sacanna, D. Bartolo, M. J. Shelley, and W. T. M. Irvine, Nat. Phys. 15, 1188 (2019).

[2] H. Li, X.-q. Shi, M. Huang, X. Chen, M. Xiao, C. Liu, H. Chaté, and H. P. Zhang, Proc. Natl. Acad. Sci. U.S.A. 116, 777 (2019).

[3] T. Sugi, H. Ito, M. Nishimura, and K. H. Nagai, Nat. Commun. 10, 683 (2019).

[4] N. Bain and D. Bartolo, Science 363, 46 (2019).

[5] G. Duclos, R. Adkins, D. Banerjee, M. S. E. Peterson, M. Varghese, I. Kolvin, A. Baskaran, R. A. Pelcovits, T. R. Powers, A. Baskaran, F. Toschi, M. F. Hagan, S. J. Streichan, V. Vitelli, D. A. Beller, and Z. Dogic, Science 367, 1120 (2020).

[6] T. Strübing, A. Khosravanizadeh, A. Vilfan, E. Bodenschatz, R. Golestanian, and I. Guido, Nano Lett. 20, 6281 (2020).

[7] A. Deblais, A. C. Maggs, D. Bonn, and S. Woutersen, Phys. Rev. Lett. 124, 208006 (2020).

[8] L. Yamauchi, T. Hayata, M. Uwamichi, T. Ozawa, and K. Kawaguchi, arXiv:2008.10852.

[9] A. Sciortino and A. R. Bausch, Proc. Natl. Acad. Sci. U.S.A. 118, e2017047118 (2021).

[10] M. Rajabi, H. Baza, T. Turiv, and O. D. Lavrentovich, Nat. Phys. 17, 260 (2021).

[11] R. Zhang, S. A. Redford, P. V. Ruijgrok, N. Kumar, A. Mozaffari, S. Zemsky, A. R. Dinner, V. Vitelli, Z. Bryant, M. L. Gardel, and J. J. de Pablo, Nat. Mater. 20, 875 (2021).

[12] E. Tjhung, C. Nardini, and M. E. Cates, Phys. Rev. X 8, 031080 (2018).

[13] Y. Baek, A. P. Solon, X. Xu, N. Nikola, and Y. Kafri, Phys. Rev. Lett. 120, 058002 (2018).

[14] B. Mahault, X.-c. Jiang, E. Bertin, Y.-q. Ma, A. Patelli, X.-q. Shi, and H. Chaté, Phys. Rev. Lett. 120, 258002 (2018).

[15] A. Souslov, K. Dasbiswas, M. Fruchart, S. Vaikuntanathan, and V. Vitelli, Phys. Rev. Lett. 122, 128001 (2019).

[16] P. Pietzonka, É. Fodor, C. Lohrmann, M. E. Cates, and U. Seifert, Phys. Rev. X 9, 041032 (2019).

[17] C. Scheibner, W. T. M. Irvine, and V. Vitelli, Phys. Rev. Lett. 125, 118001 (2020).

[18] S. Saha, J. Agudo-Canalejo, and R. Golestanian, Phys. Rev. X 10, 041009 (2020).

[19] L. P. Dadhichi, J. Kethapelli, R. Chajwa, S. Ramaswamy, and A. Maitra, Phys. Rev. E 101, 052601 (2020).

[20] R. Zakine, Y. Zhao, M. Knežević, A. Daerr, Y. Kafri, J. Tailleur, and F. van Wijland, Phys. Rev. Lett. 124, 248003 (2020).

[21] Z. You, A. Baskaran, and M. C. Marchetti, Proc. Natl. Acad. Sci. U.S.A. 117, 19767 (2020). 
[22] D. Banerjee, V. Vitelli, F. Jülicher, and P. Surówka, Phys. Rev. Lett. 126, 138001 (2021).

[23] J. Denk and E. Frey, Proc. Natl. Acad. Sci. U.S.A. 117, 31623 (2020).

[24] F. Meng, D. Matsunaga, B. Mahault, and R. Golestanian, Phys. Rev. Lett. 126, 078001 (2021).

[25] C. Reichhardt and C. J. O. Reichhardt, Phys. Rev. E 103, 022602 (2021).

[26] J. Toner and Y. Tu, Phys. Rev. Lett. 75, 4326 (1995).

[27] J. Toner and Y. Tu, Phys. Rev. E 58, 4828 (1998).

[28] Y. Tu, J. Toner, and M. Ulm, Phys. Rev. Lett. 80, 4819 (1998).

[29] J. Toner, Phys. Rev. E 86, 031918 (2012).

[30] J. Toner, Phys. Rev. Lett. 108, 088102 (2012).

[31] B. Mahault, F. Ginelli, and H. Chaté, Phys. Rev. Lett. 123, 218001 (2019).

[32] L. Chen, C. F. Lee, and J. Toner, Nat. Commun. 7, 12215 (2016).

[33] A. Maitra, P. Srivastava, M. C. Marchetti, S. Ramaswamy, and M. Lenz, Phys. Rev. Lett. 124, 028002 (2020).

[34] H. Chaté, Annu. Rev. Condens. Matter Phys. 11, 189 (2020).

[35] The only exception we are aware of is [36] but the coupling of the $2 \mathrm{D}$ active layer to a surrounding $3 \mathrm{D}$ fluid is essential to insure long-range order.

[36] A. Maitra, arXiv:2010.15044.

[37] R. A. Simha and S. Ramaswamy, Phys. Rev. Lett. 89, 058101 (2002).

[38] S. Ramaswamy, R. A. Simha, and J. Toner, Europhys. Lett. 62, 196 (2003).

[39] J. Toner, Y. Tu, and S. Ramaswamy, Ann. Phys. (Amsterdam) 318, 170 (2005).

[40] S. Mishra, R. A. Simha, and S. Ramaswamy, J. Stat. Mech. (2010) P02003.

[41] M. C. Marchetti, J.-F. Joanny, S. Ramaswamy, T. B. Liverpool, J. Prost, M. Rao, and R. A. Simha, Rev. Mod. Phys. 85, 1143 (2013).
[42] S. Shankar, S. Ramaswamy, and M. C. Marchetti, Phys. Rev. E 97, 012707 (2018).

[43] A. Maitra, P. Srivastava, M. C. Marchetti, J. S. Lintuvuori, S. Ramaswamy, and M. Lenz, Proc. Natl. Acad. Sci. U.S.A. 115, 6934 (2018).

[44] F. Ginelli, F. Peruani, M. Bär, and H. Chaté, Phys. Rev. Lett. 104, 184502 (2010).

[45] D. Nishiguchi, K. H. Nagai, H. Chaté, and M. Sano, Phys. Rev. E 95, 020601(R) (2017).

[46] See Supplemental Material at http://link.aps.org/ supplemental/10.1103/PhysRevLett.127.048003 for details about numerical methods and analytical calculations, which includes Ref. [47].

[47] L.-b. Cai, H. Chaté, Y.-q. Ma, and X.-q. Shi, Phys. Rev. E 99, 010601(R) (2019).

[48] $\ell_{r}$ (thus $\tau_{r}$ ) would in general depend on $\rho_{0}$ and $\eta$, but as our analysis remains qualitative we do not need to specify its exact form.

[49] E. Bertin, H. Chaté, F. Ginelli, S. Mishra, A. Peshkov, and S. Ramaswamy, New J. Phys. 15, 085032 (2013).

[50] A. Peshkov, E. Bertin, F. Ginelli, and H. Chaté, Eur. Phys. J. Spec. Top. 223, 1315 (2014).

[51] H. Chaté and B. Mahault, in Active Matter and NonEquilibrium Statistical Physics: A Synthetic and SelfContained Overview, edited by J. Tailleur (Oxford University Press, New York, 2019), Chap. Dilute Dry Aligning Active Matter.

[52] Note that this propagative ansatz is at odds with the diffusive one usually at play in active nematics.

[53] Choosing $\bar{g}$ real assumes, with loss of generality, that order is along $x$.

[54] We included in Eq. (4b) the term $D_{0 \perp} \partial_{\perp \perp}^{2} \delta g_{0}$, allowed by symmetries, even though it does not appear in the derivation.

[55] B. Mahault and H. Chaté (to be published).

[56] A simple argument shows that $\varpi=-2 \chi / \xi$, see [46]. 